\title{
Polarization analysis of a Pi2 pulsation using continuous wavelet transform
}

\author{
M. Kulesh ${ }^{1}$, M. Nosé2, M. Holschneider ${ }^{1}$, and K. Yumoto ${ }^{3}$ \\ ${ }^{1}$ Institute for Mathematics, University of Potsdam, Am Neuen Palais 10, 14469 Potsdam, Germany \\ ${ }^{2}$ Data Analysis Center for Geomagnetism and Space Magnetism, Kyoto University, \\ Kitashirakawa Oiwake-cho, Sakyo-ku, Kyoto 606-8502, Japan \\ ${ }^{3}$ Space Environment Research Center, Kyushu University, 6-10-1 Hakozaki, Fukuoka 812-8581, Japan
}

(Received December 4, 2006; Revised May 21, 2007; Accepted May 31, 2007; Online published August 31, 2007)

\begin{abstract}
In this contribution, we extend a series of previous works focused on an investigation of signal's polarization attributes using the continuous wavelet transform, where we proposed a method to map instantaneous polarization attributes of multicomponent signals in the wavelet domain and explicitly relate these attributes with the wavelet transform coefficients of the analyzed signal. In this work, we applied our polarization method to an examination of characteristics of $\mathrm{Pi} 2$ pulsations. We have shown some merits of the use of the continuous wavelet transform for the Pi2 pulsations' analysis. First, we used our polarization method for the geomagnetic field data from the MSR, KAK, GUA, SMA, BLM and LAQ observatories and showed some correlations between the polarization parameters of pulsation and the station's position (nightside or dayside). Secondly, we considered the signal's north components of a pair of stations and demonstrated a time-frequency variations of the phase difference between two stations during the pulsation.
\end{abstract}

Key words: Polarization attributes, multicomponent signal, wavelet transform, Pi2 pulsation.

\section{Introduction}

$\mathrm{Pi} 2$ pulsations are defined as damped oscillations of the geomagnetic field with the period range of 40-150 s. This kind of pulsations can be observed clearly at low- and midlatitude ground stations at substorm onset. Recent studies (Nosé et al., 2003, 2006) have shown that low-latitude Pi2 pulsations can be found at multiple stations which are longitudinally separated by a large distance of about 5-11 h of magnetic local time (MLT). These researchers found out that most of Pi2 pulsations does not have any phase differences among the stations, implying that the longitudinal wave number of pulsations $(\mathrm{m})$ is close to zero. However, they also found a small number of Pi2 pulsations which have small phase differences corresponding to $|m| \sim 0.24$ 0.48. Kosaka et al. (2002) and Han et al. (2003) reported that the dominant frequency of mid- and low-latitude Pi2 pulsations depends on the local time. It is therefore important to examine the longitudinal dependence of low-latitude Pi2 pulsations' characteristics (i.e. phase difference or frequency), since this provides information to reveal the excitation mechanism of $\mathrm{Pi} 2$ pulsations.

Fourier analysis is a traditional tool by which to analyze a time series. It has been used to determine the dominant frequency of a signal as well as the phase difference between two signals. However, it is difficult for Fourier analysis to detect changes of dominant frequency or phase difference within a short time, such as few wave cycles.

Frequency-dependent measurements or time-frequency analysis are particularly suitable to resolve these time-

Copyright (c) The Society of Geomagnetism and Earth, Planetary and Space Sciences (SGEPSS); The Seismological Society of Japan; The Volcanological Society of Japan; The Geodetic Society of Japan; The Japanese Society for Planetary Sciences; TERRAPUB. dependent properties. This analysis consists of an examination of the frequency content variation of a signal over time. For the extended analysis of two- or three-component signals, the time-frequency representation can be incorporated into the polarization analysis (Pinnegar, 2006; Diallo et al., 2006). Several methods are proposed in the literature for polarization analysis of multicomponent data. Some of these methods allow the polarization attributes to be determined directly from the multicomponent signal (Morozov and Smithson, 1996; Rene et al., 1986), others are based on the analysis of the covariance matrix of multicomponent recordings and principal component analysis using the singular value decomposition (Kanasewich, 1981).

In our previous work (Diallo et al., 2006), we proposed a new method of polarization analysis based on the continuous wavelet transform, which enables the computation of all polarization properties and, in particular, the instantaneous frequency and the instantaneous phase difference. In this paper, we apply this new method to some of $\mathrm{Pi} 2$ pulsation events and investigate how the phase difference of a signal is changing within a short time.

The continuous wavelet used in our approach is not critical for understanding the presented results. Other methods of time-frequency analysis, such as the Gabor transform or the S-transform, can be generally used to obtain the polarization properties. The relative performance of polarization analysis from this different approach is primarily controlled by the frequency resolution capability. Particularly, the short-time Fourier transform or the typical dynamic spectrum can be very helpful in the analysis of geomagnetic pulsations in the case of the spectral resolution being important. However, the main subject in this paper is the observation of the time variations of polarization prop- 
erties for certain frequencies and, therefore, the time resolution grows in importance in additional to spectral resolution. The continuous wavelet transform gives a suitable general framework for solving this type of problem because we can control the time or spectral resolution capability by the choice of an appropriate wavelet.

This paper is organized as follows. In the following section, we give an overview of the continuous wavelet transform. We then briefly introduce the new method to calculate polarization properties of two-component signals in the time-frequency domain. This new method will be applied to the geomagnetic field data, and the results of this analysis will be presented in Section 4. The last section presents conclusions.

\section{Introduction to the Continuous Wavelet Trans- form}

At least two different perspectives exist for wavelet analysis. The first one is the interpretation of the wavelet transform as a time-frequency analysis tool. The second approach uses the wavelet analysis as mathematical microscope, and is therefore closely linked to approximation theory (Holschneider, 1995). Our present work focuses on the first approach of wavelet analysis.

The continuous wavelet transform of a real or complex signal $S(t) \in L^{2}(\mathbb{R})$ with respect to a mother wavelet $g(t)$ is the set of $L^{2}$-scalar products of all dilated and translated wavelets with an arbitrary signal to be analyzed:

$$
\begin{array}{r}
\mathcal{W}_{g} S(t, a)=\left\langle g_{t, a}, S\right\rangle=\int_{-\infty}^{+\infty} \frac{1}{a} g^{*}\left(\frac{\tau-t}{a}\right) S(\tau) d \tau \\
a \in \mathbb{R}, t \in \mathbb{R},
\end{array}
$$

where $g_{t, a}(\tau)=\frac{1}{a} g((\tau-t) / a)$ is generated from $g(\tau)$ through dilation $a$ and translation $t$. The symbol (.)* denotes the complex conjugate. The wavelet $g(t)$ is assumed to be a function which is well localized in time and frequency and obeys the oscillation condition $\int_{-\infty}^{+\infty} g(t) d t=$ 0 .

The signal $S(t)$ can be recovered from its wavelet transform as

$$
\begin{aligned}
S(t) & =\mathcal{M}_{m} \mathcal{W}_{g} S(\tau, a) \\
& =\frac{1}{C_{g, m}} \int_{-\infty}^{\infty} \int_{-\infty}^{+\infty} \frac{1}{a^{2}} m\left(\frac{t-\tau}{a}\right) \mathcal{W}_{g} S(\tau, a) d \tau d a, \\
C_{g, m} & =\int_{0}^{+\infty}\left(\hat{g}^{*}(\omega) \hat{m}(\omega)+\hat{g}^{*}(-\omega) \hat{m}(-\omega)\right) \frac{d \omega}{\omega},
\end{aligned}
$$

where $m(t)$ is the wavelet used for the inverse wavelet transform $\mathcal{M}_{m}$, and $\omega$ is the angular frequency. In the general case, the wavelet $m(t)$ can be different from the direct transform wavelet $g(t)$.

In the particular case, for the inverse wavelet transform we might choose the $\delta$-function for the wavelet $m(t)$, which then gives us the rather simple reconstruction formula

$$
S(t)=\frac{1}{C_{g, \delta}} \int_{-\infty}^{+\infty} \mathcal{W}_{g} S(t, a) \frac{d a}{a} .
$$

The wavelet transform can be expressed in terms of the Fourier transform $\hat{S}(\omega)$ of $S(t)$ as

$$
\mathcal{W}_{g} S(t, a)=\int_{-\infty}^{+\infty} \hat{g}^{*}(a \zeta) e^{2 \pi i t \zeta} \hat{S}(\zeta) d \zeta,
$$

and the inverse of the scale $1 / a$ may be associated with a frequency measured in units of the central frequency of $g(t)$. If the central frequency of the wavelet is assumed to be $f_{0}$, each scale $a$ can be related to the physical frequency $f$ by $a=f_{0} / f$. Therefore, if we select a wavelet with a unitcentral frequency, it can be possible to obtain the physical frequency directly by taking the inverse of the scale.

The choice of the analyzing wavelet depends on what typical oscillation modes are present in the signal. For example, to analyze rectangular oscillations, the real HAAR wavelet or a similar but symmetric FHAT wavelet is most suitable. If only one component of geomagnetic signals is analyzed, any real wavelets based on different-order derivatives of the Gaussian are convenient. However, the method of polarization analysis proposed in the next sections is based on a complex wavelet.

We also will need later a feature of the wavelet transform which allows us to separate the wavelet spectrum into progressive and regressive components,

$$
\mathcal{W}_{g} S(t, f)=\mathcal{W}_{g}^{+} S(t, f)+\mathcal{W}_{g}^{-} S(t, f),
$$

where

$$
\mathcal{W}_{g}^{+} S(t, f)=\left\{\begin{array}{l}
\mathcal{W}_{g} S(t, f), \quad f \geq 0 \\
0, \quad f<0
\end{array}\right.
$$

and

$$
\mathcal{W}_{g}^{-} S(t, f)=\left\{\begin{array}{l}
0, \quad f \geq 0 \\
\mathcal{W}_{g} S(t, f), \quad f<0
\end{array}\right.
$$

To perform such a separation for nonprogressive wavelets, one must first calculate the Hilbert transform of the source signal, which makes the numerical procedure more complex. Therefore, we use here a complex progressive wavelet, which has zero Fourier coefficients for negative frequencies.

For example, we can consider the widely used complex Morlet wavelet consisting of a plane wave modulated by a Gaussian

$$
g(t)=e^{2 \pi i t} e^{-t^{2} /\left(2 \sigma^{2}\right)}, \quad \hat{g}(\omega)=\sigma \sqrt{2 \pi} e^{-\sigma^{2}(\omega-2 \pi)^{2} / 2},
$$

where $\hat{g}(\omega)$ denotes the Fourier transform of the wavelet $g(t)$ and $\sigma$ describes the variance of the wavelet. The Fourier transform of this wavelet is real-valued. Strictly speaking, the Morlet wavelet is neither progressive or regressive nor admissible but can be considered to be numerically equivalent to a progressive or regressive wavelet when $\sigma>5$.

We also can similarly use the complex Cauchy wavelet of power $p$ (Fig. 1). This wavelet can be written as

$$
\begin{aligned}
& g(t)=\Gamma(p)\left(1-\frac{2 \pi i t}{p-1}\right)^{-p}, \\
& \hat{g}(\omega)=(\omega / 2 \pi)^{p-1} e^{-\omega(p-1) / 2 \pi},
\end{aligned}
$$

where $\Gamma(p)$ is the Euler gamma function. 

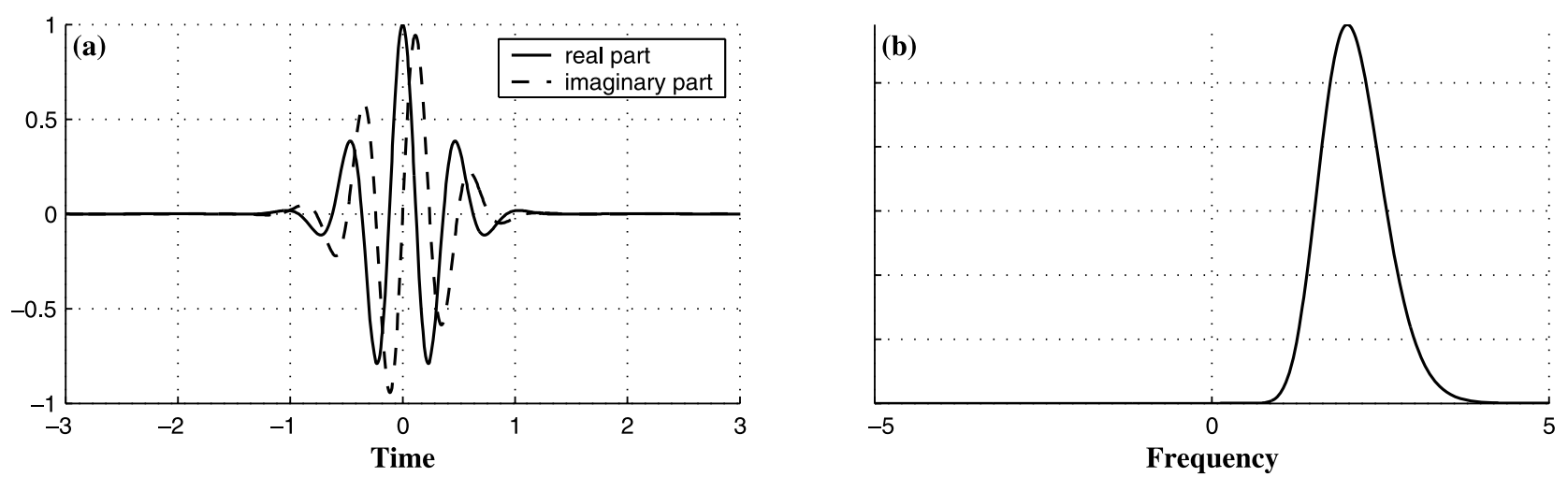

Fig. 1. An example of complex Cauchy wavelet in (a) time domain and (b) frequency domain.

(a)

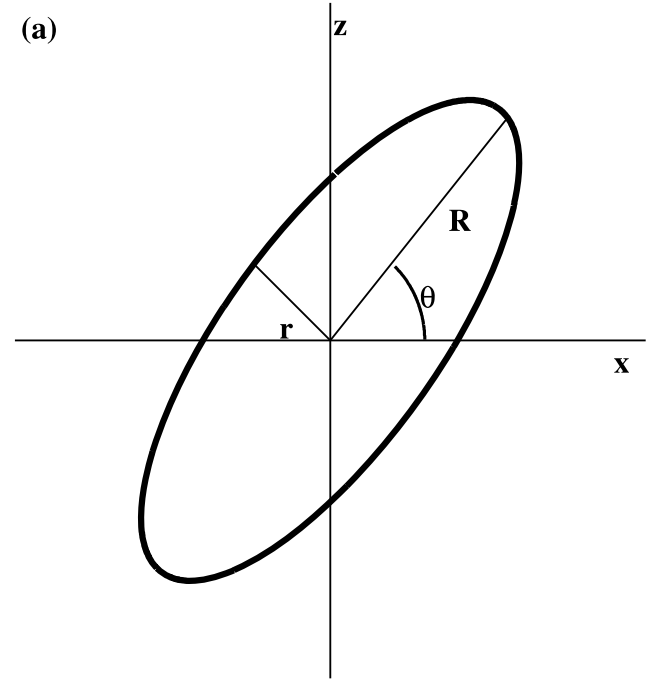

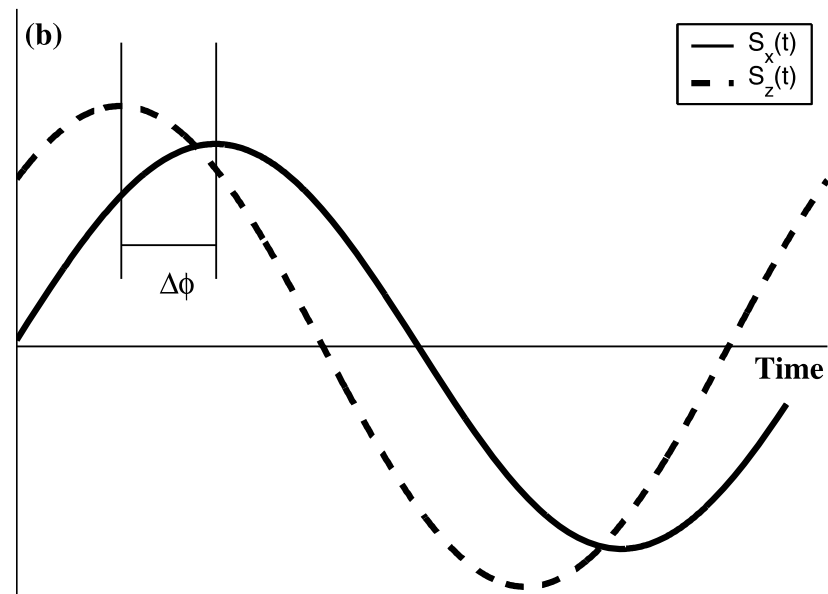

Fig. 2. Schematic representation of an ellipse with its geometric parameters: the semi-major axis $R$, the semi-minor axis $r$, the tilt angle $\Theta$ and the phase difference $\Delta \phi$ between $S_{x}(t)$ and $S_{z}(t)$. The components $x$ and $z$ are related to the north and east components of magnetic data respectively.

\section{Instantaneous Polarization Attributes in the Wavelet Domain}

Rene et al. (1986) proposed a method for multicomponent analysis based on complex trace analysis (CTA). This method allows the computation of instantaneous polarization attributes. However, the CTA-based method operates only in the time domain, and the estimated attributes represent an average over all frequencies and therefore do not provide information on their frequency dependency. To cope with these limitations inherent to time-frequency resolution, we use here a method based on the continuous wavelet transform proposed by Diallo et al. (2006). First, we briefly introduce this method.

Our goal is to derive instantaneous polarization attributes from two signals, $S_{\text {north }}(t)$ and $S_{\text {east }}(t)$, corresponding to the north and east components of magnetic data, respectively. Using these components, we construct a complex signal

$$
Z(t)=S_{\text {north }}(t)+i S_{\text {east }}(t) .
$$

In its full generality, an elliptically polarized rotating signal $Z(t)$ is described by following geometric parameters (Fig. 2):

1) $R$ : the semi-major axis $R \geq 0$,
2) $r$ : the semi-minor axis $r \geq 0$,

3) $\rho=r / R$ : the ellipticity ratio, $\rho \in[0,1]$,

4) $\Theta$ : the tilt angle, which is the angle of the semi-major axis with the horizontal axis, $\Theta \in[-\pi / 2, \pi / 2]$, and

5) $\Delta \phi$ : the phase difference between $S_{\text {north }}(t)$ and $S_{\text {east }}(t)$ components.

The key idea to obtain instantaneous polarization attributes is the approximation of the complex signal $Z(t)$ at the time point $t$ by a turning ellipse $C(\tau)$ on the complex plane (Fig. 2(a)). The most general turning ellipse is parameterized by two complex numbers, $A^{+}$and $A^{-}$and two real numbers $\omega^{+}$and $\omega^{-}$:

$$
C(\tau)=A^{+} e^{i \omega^{+} \tau}+A^{-} e^{-i \omega^{-} \tau} .
$$

This formula, along with geometrical considerations, yields the following relationships:

$$
\begin{aligned}
& R=\left|A^{+}\right|+\left|A^{-}\right|, \quad r=|| A^{+}|-| A^{-}||, \\
& \Theta=\frac{1}{2} \arg \left(A^{+} A^{-}\right), \\
& \Delta \phi=\arg \left(\frac{A^{+}+\left(A^{-}\right)^{*}}{A^{+}-\left(A^{-}\right)^{*}}\right)+\frac{\pi}{2} .
\end{aligned}
$$

When a complex progressive wavelet is used, the prop- 


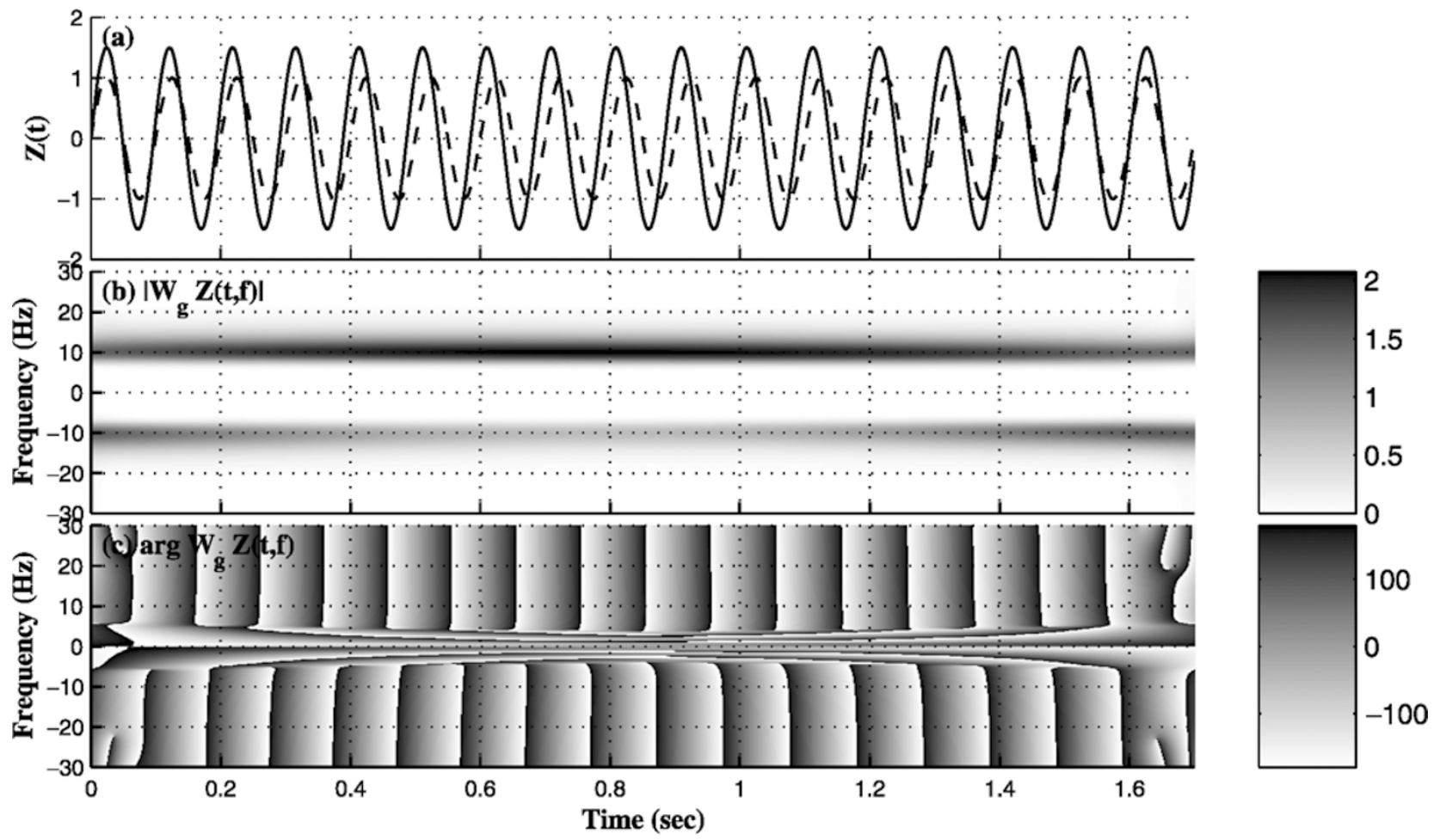

Fig. 3. Time-frequency spectrum of the complex synthetic signal: (a) north (solid line) and east (dotted line) components of the signal, (b) the modulus and (c) the phase of the wavelet spectrum.
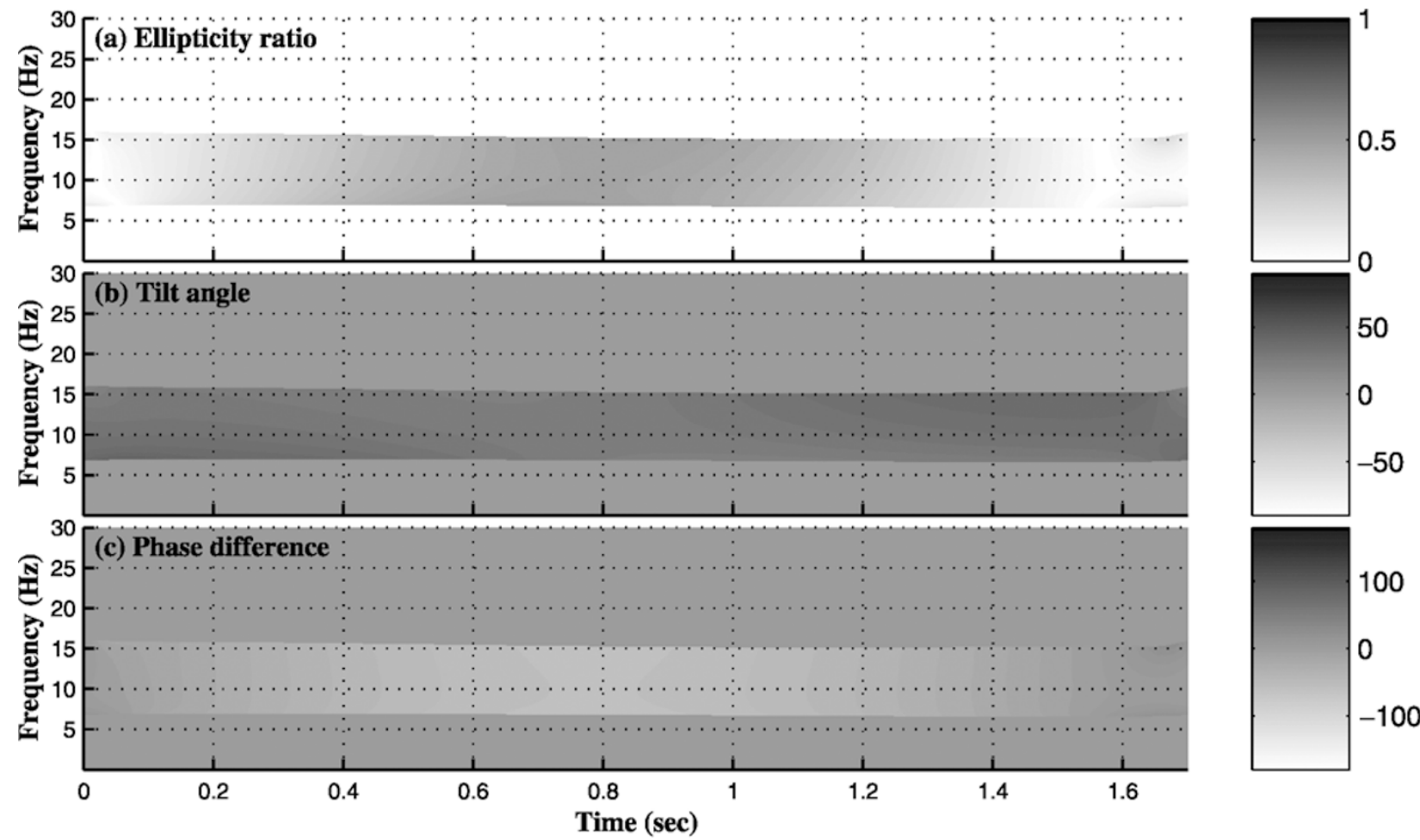

Fig. 4. Time-frequency polarization spectrum of the complex synthetic signal: (a) the ellipticity ratio, (b) the tilt angle and (c) the phase difference between $S_{\text {north }}(t)$ and $S_{\text {east }}(t)$ components.

erty (3) of the wavelet transform allows us to represent the wavelet spectrum as a superposition of its progressive and regressive components. Let us consider the instantaneous angular frequency defined as the derivative of the complex spectrum's phase: $\omega^{ \pm}(t, f)= \pm \partial \arg \mathcal{W}_{g}^{ \pm} Z(t, f) / \partial t$,
Then, near time instant $t$, each component can be represented as follows:

$$
\begin{aligned}
\mathcal{W}_{g} Z(t+\tau, f) \simeq & \mathcal{W}_{g}^{+} Z(t, f) e^{i \omega^{+}(t, f) \tau} \\
& +\mathcal{W}_{g}^{-} Z(t, f) e^{-i \omega^{-}(t, f) \tau}
\end{aligned}
$$


which yields the time-frequency spectrum for each of the parameters:

$$
\begin{aligned}
& R(t, f)=\left|\mathcal{W}_{g}^{+} Z(t, f)\right|+\left|\mathcal{W}_{g}^{-} Z(t, f)\right|, \\
& r(t, f)=|| \mathcal{W}_{g}^{+} Z(t, f)|-| \mathcal{W}_{g}^{-} Z(t, f) \|, \\
& \rho(t, f)=r(t, f) / R(t, f) \\
& \Theta(t, f)=\arg \left[\mathcal{W}_{g}^{+} Z(t, f) \mathcal{W}_{g}^{-} Z(t, f)\right] / 2, \\
& \Delta \phi(t, f)=\arg \left(\frac{\mathcal{W}_{g}^{+} Z(t, f)+\left(\mathcal{W}_{g}^{-} Z(t, f)\right)^{*}}{\mathcal{W}_{g}^{+} Z(t, f)-\left(\mathcal{W}_{g}^{-} Z(t, f)\right)^{*}}\right)+\frac{\pi}{2} .(7)
\end{aligned}
$$

To demonstrate these instantaneous properties, let us consider a synthetic example. In the next section, we will apply polarization method (7) to the investigation of phase differences in the geomagnetic records; therefore, we consider as an example a sinusoidal waveform with a single frequency component, constant amplitudes, and smooth phase changes. This signal is not based on a physical model; our aim is to show the time-frequency resolution of our method with reference to the analysis of the phase difference. We define the complex synthetic signal having a small phase difference between its two components as follows:

$$
\begin{aligned}
& Z(t)=S_{\text {north }}(t)+i S_{\text {east }}(t), \\
& S_{\text {north }}(t)=R \sin \left(2 \pi f_{0} t+\sin f_{1} t\right), \\
& S_{\text {east }}(t)=r \sin \left(2 \pi f_{0} t\right),
\end{aligned}
$$

where $f_{0}=10 \mathrm{~Hz}, R=1.5, r=1$, and $f_{1}=2$. This signal is shown in Fig. 3(a).

We choose the Cauchy wavelet with parameter $p=20$ (see Eq. (5)) and apply the continuous wavelet transform introduced by Eqs. (1) or (2) for the frequency range from -30 to $30 \mathrm{~Hz}$. It allows us to obtain the full wavelet spectrum $\mathcal{W}_{g} Z(t, f)$ that includes both regressive $\mathcal{W}_{g}^{-} Z(t, f)$ and progressive $\mathcal{W}_{g}^{+} Z(t, f)$ parts (see Eq. (3)). Both parts of the wavelet spectrum are shown in Figs. 3(b, c), where $\mathcal{W}_{g}^{+} Z(t, f)$ corresponds to positive frequencies and $\mathcal{W}_{g}^{-} Z(t, f)$ to negative.

The analysis of the differences between progressive and regressive parts allows us to calculate all elliptic properties in the corresponding time-frequency area. For such a calculation, we use Eqs. (7) and obtain the ellipticity ratio $\rho(t, f)$, the tilt angle $\Theta(t, f)$ and the phase difference $\Delta \phi(t, f)$ between the $S_{\text {north }}(t)$ and $S_{\text {east }}(t)$ components as a time-frequency spectrum for the frequency range from 0.001 to $30 \mathrm{~Hz}$. However, the frequency areas where the modulus $\left|\mathcal{W}_{g} Z(t, f)\right|$ of the wavelet spectrum (Fig. 3(b)) is close to zero do not contain any helpful information about the signal structure; therefore it is defensible to cut-off the elliptical parameters. For example, it can be done using this modulus and a threshold parameter $\varepsilon$ as follows:

$$
\begin{aligned}
& \rho(t, f)= \\
& \begin{cases}\rho(t, f), & \\
\left|\mathcal{W}_{g}^{+} Z(t, f)\right|+\left|\mathcal{W}_{g}^{-} Z(t, f)\right|> & \varepsilon \cdot \max \left(\left|\mathcal{W}_{g}^{+} Z(t, f)\right|\right. \\
0, & \left.+\left|\mathcal{W}_{g}^{-} Z(t, f)\right|\right), \\
\text { otherwise. }\end{cases}
\end{aligned}
$$

Because our synthetic signal is a pure harmonic function with central frequency $f_{0}=10 \mathrm{~Hz}$, all energy content of this signal is concentrated in the narrow frequency band from 7 to $15 \mathrm{~Hz}$, which is indicated in the pictures as narrow regions over the full time interval. To plot all polarization properties (Fig. 4), we use the threshold parameter $\varepsilon=0.6$ that corresponds to this frequency band and causes discontinuities at $f=7 \mathrm{~Hz}$ and $f=15 \mathrm{~Hz}$. Besides, the ellipticity ratio and the phase difference show small variations from zero to the maximum and back to zero, and the tilt angle does not have any variations. This behavior corresponds with the analytical expression of this signal.

From Fig. 3(a) we can see that the phase difference between the signal components at the beginning (up to 0.4 $\mathrm{sec}$ ) and at the end (after $1.4 \mathrm{sec}$ ) of record is small; therefore, we do not see it distinctly in the signal representation. However, in Fig. 4(c) we can see it very clearly because of the distinction between background color that indicates zero phase difference and color in the frequency band $f=7 \ldots 15 \mathrm{~Hz}$ corresponding to the signal. Moreover, the color change in this frequency band indicates how the phase difference changes with time. Therefore, the proposed method allows us to "strengthen" the phase difference using a time-frequency representation.

A positive phase difference in the right-handed coordinate system corresponds to right-handed polarization, and negative phase difference, to the left-handed. However, by the signal construction given by Eq. (6) we assume that the $x$-axis is directed to the north and the $z$-axis is directed to the east, which defines a left-handed coordinate system. Therefore, we can interpret the positive phase difference as left-handed polarization of the signal. The phase difference shown in Fig. 4(c) is negavite, which is typical for signals with right-handed polarisation like our synthetic signal.

\section{Application to Real Data}

\subsection{The 20 September 1995 event}

At 0538 UT on 20 September 1995, Pi2 pulsations were observed clearly at three different MLT sectors. At the post-midnight sector $(\sim 0200 \mathrm{MLT})$, the low-latitude station SMA (Santa Maria, $-19.5^{\circ}$ geomagnetic latitude (GMLAT)) and the equatorial station BLM (Belem, 8.6 GMLAT) observed clear Pi2 pulsations. On the dawnside ( $\sim 0700 \mathrm{MLT}), \mathrm{Pi} 2$ pulsation was detected at the midlatitude station LAQ (Laquila, 42.5 $5^{\circ}$ GMLAT). Even in the afternoon sector at $\sim 1500 \mathrm{MLT}, \mathrm{Pi} 2$ pulsations were found at low-latitude stations MSR (Moshiri, $35.4^{\circ}$ GMLAT) and KAK (Kakioka, 26.9 $9^{\circ}$ GLAT) and at the equatorial station GUA (Guam, 5.1 ${ }^{\circ}$ GMLAT). This event was reported by Nosé et al. (2003) who suggested that the substorm was initiated at pre-midnight (2100-2300 MLT) by using highlatitude ground magnetic field data.

Geomagnetic field data in the northward and eastward components recorded at MSR, GUA, SMA, BLM and LAQ are presented on the left panel of Fig. 5. On the right panel of Fig. 5, the results of the polarization analysis of the geomagnetic field data are displayed. Gray-scaled images show the time-frequency variation of the phase difference $\Delta \phi(t, f)$ calculated using Eq. (7). Displaying any attribute in the time-frequency space would primarily serve to identify coherent structures that belong to the same geomagnetic event. 

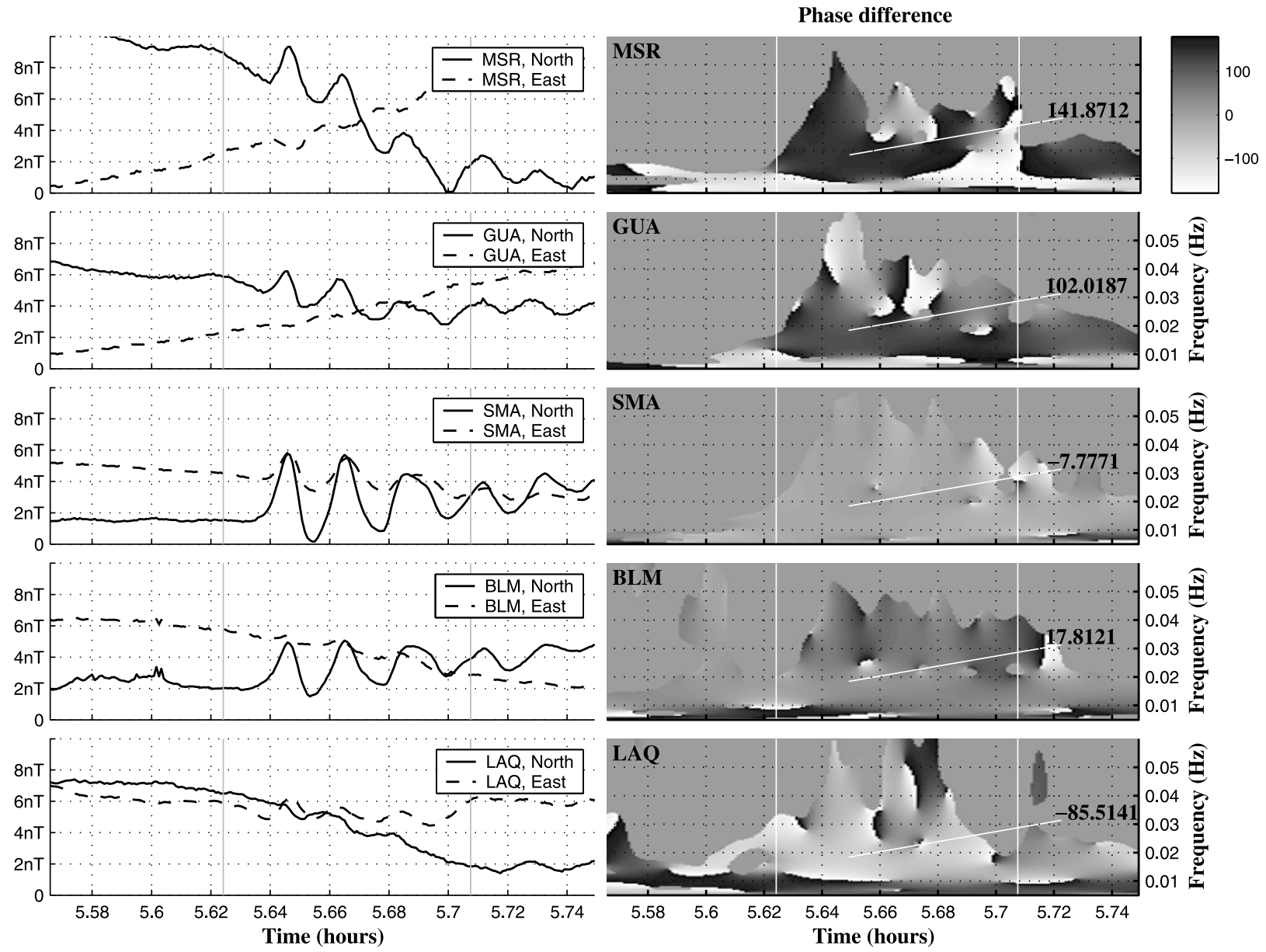

Fig. 5. Source signals and time-frequency representations of phase differences. Vertical lines bound the interval of Pi2 pulsation.

From gray-scaled images it is difficult to evaluate the exact numerical values of the parameters, therefore, we also showed on these images an example of the phase difference values (in degrees) at the time point $t=5.65 \mathrm{~h}$ and by the frequency $f=0.018 \mathrm{~Hz}$. However, the value of phase difference in some detached points of time-frequency domain cannot be used for any physical interpretations. More suitable for such an interpretation is the analysis of the average of $\Delta \phi(t, f)$ either along the time or the frequency parameter.

One manner to reconstruct frequency-dependent quantities is to average the above formulas (7) over a suitable time region in which a Pi2 has been identified in wavelet space:

$$
\Delta \phi(f)=\frac{1}{\Delta T} \int_{T_{1}}^{T_{2}} \Delta \phi(\tau, f) d \tau .
$$

In Fig. 6, we show an example of frequency-dependent phase differences for all considered stations. SMA and BLM stations are characterized by almost zero phase difference, indicating linear polarization. Previous studies have shown that Pi2 on the nightside has left-handed or linear polarization in general (Lester et al., 1983, 1984; Lanzerotti and Medford, 1984). Thus, our results are consistent with this. MSR and GUA, which were located around 1500 MLT, have positive phase difference in the interval $100^{\circ}-$ $140^{\circ}$, with respect to the left-handed polarization. However,
Pi2 pulsation observed at LAQ around 0700 MLT had a negative phase difference of $-80^{\circ}$. Nosé (1999) reported that $\mathrm{Pi} 2$ pulsations have dayside polarization characteristics different from nightside $\mathrm{Pi} 2$ pulsations, being right-handed before local noon and changes to left-handed after local noon. Polarization of dayside Pi2 pulsations shown in Fig. 6 for LAQ station is consistent with that reported by Nosé (1999).

Figure 7 shows the ellipticity ratio and tilt angle in the time-frequency domain for $\mathrm{Pi} 2$ pulsations presented on the left panel of Fig. 5. The ellipticity ratios at MSR, GUA, SMA, and BLM are small, which indicates that oscillations are dominant the direction defined by tilt angle. This is consistent with results reported by Yumoto et al. (1994) and Osaki et al. (1996). Ellipticity at LAQ seems larger than that at the other four stations, implying that oscillations in the northward direction are smaller. This would be caused by the fact that LAQ was located on the dawnside, where the power spectral density of $\mathrm{Pi} 2$ pulsations in the northward component becomes weak (Nosé, 1999).

The right panel of Fig. 7 shows that SMA had positive tilt angle, while MSR had negative tilt angles. The tilt angles at GUA and BLM, which are located near the geomagnetic equator, were close to $0^{\circ}$. This indicates that the major polarization axis of Pi2 pulsations at SMA was oriented to the north-east and that at MSR was oriented to the north-west. GUA and BLM showed the major polarization axis to be almost along the north-south direction. The tilt angle at LAQ 


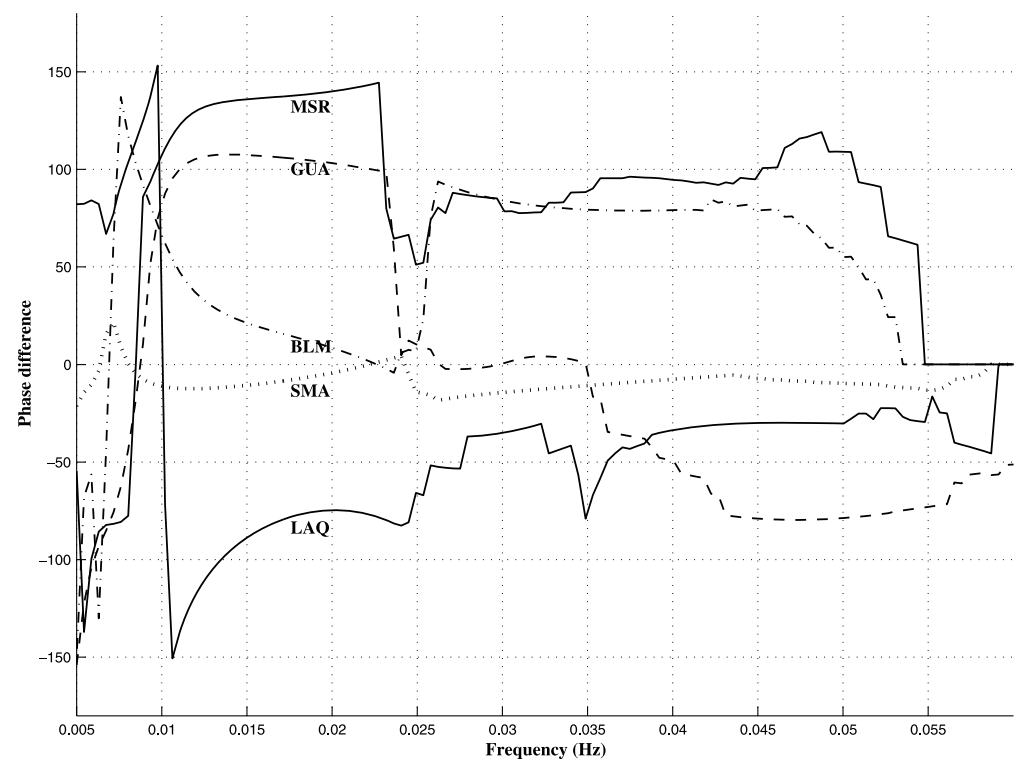

Fig. 6. Frequency-dependent phase differencies $\Delta \phi(f)$ averaged in the time interval between $5.64 \mathrm{~h}$ and $5.66 \mathrm{~h}$.
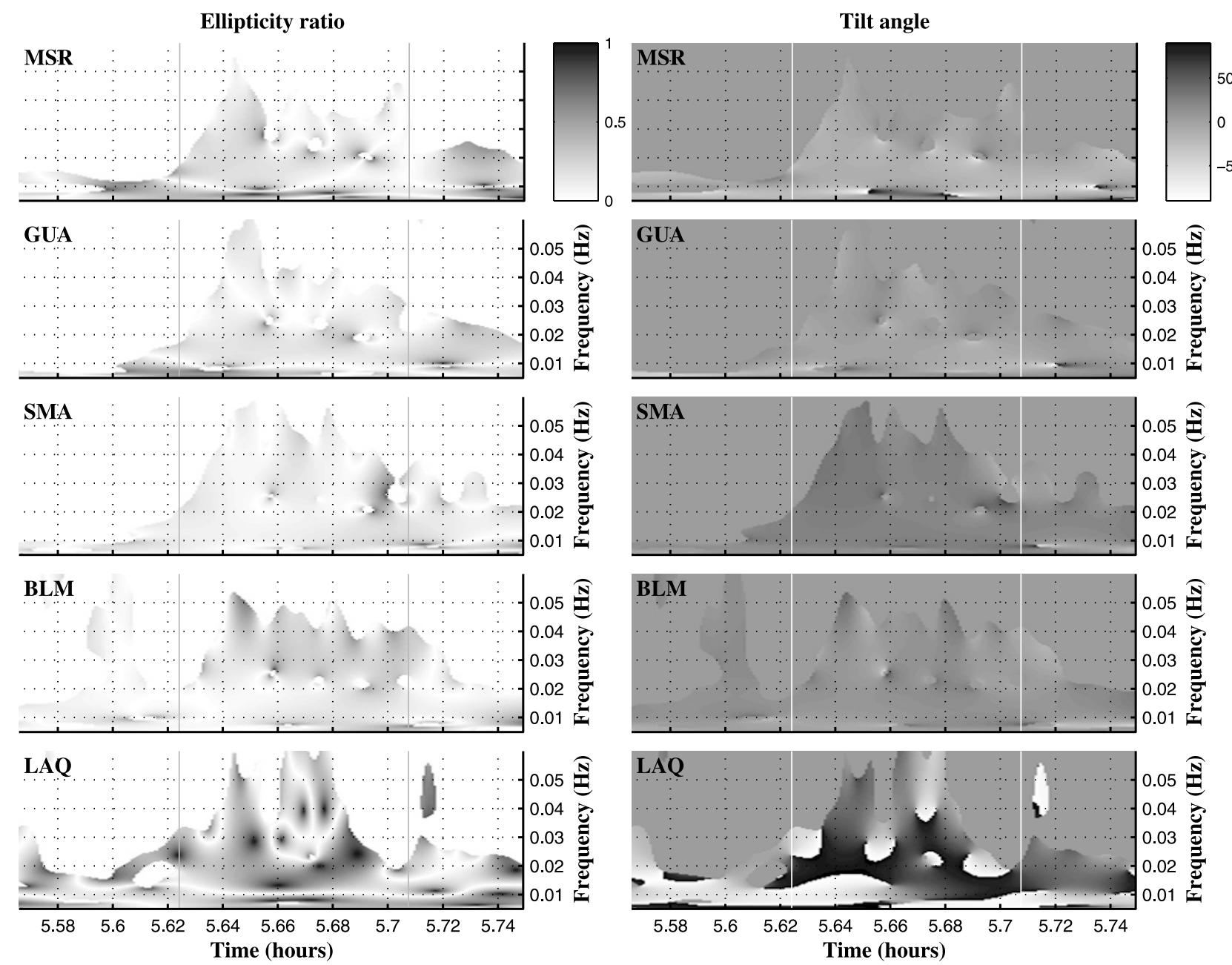

Fig. 7. Ellipticity ratio and tilt angle in time-frequency domain for signals shown in Fig. 5.

was more variable than that other stations and was as large as $\simeq \pm 90^{\circ}$. These results suggest that the orientation of the polarization axis is different between equatorial stations and low-latitude stations.
Figure 8 shows the phase difference of the geomagnetic field in the northward direction between different stations. From the left panels of this figure we denote the presence of phase differences (for example, obvious component shift 

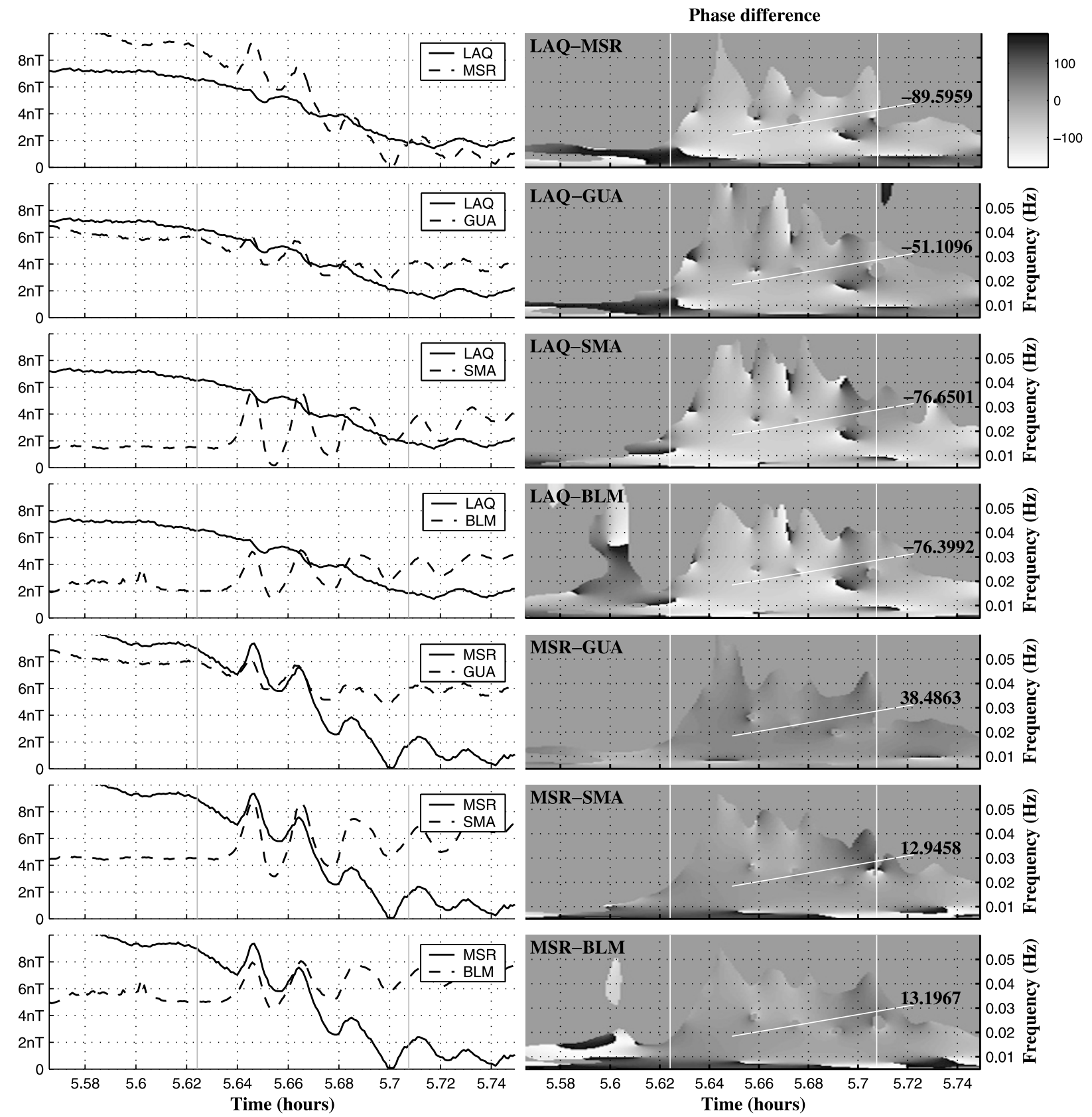

Fig. 8. Northward components of source signals registered at different stations and time-frequency phase difference between the pair of stations.

for MSR-GUA stations), while the numerical value of this shift we can read from the right panels of the same figure. Note that the phase differences for first five examples do not depend on the time and frequency; therefore, we can interpret the given numerical values as mean phase differences for whole event. At the same time, two last examples, MSRSMA and MSR-BLM show some variations of the phase difference, with the time-frequency area restricting the pulsation, and we cannot directly interpret the numerical values as mean values.

From this figure we found that the phase difference is about $-75^{\circ}$ for the LAQ-SMA and LAQ-BLM pairs, about $-90^{\circ}$ for the LAQ-MSR pair, $-50^{\circ}$ for the LAQ-GUA pair and about $40^{\circ}$ for MSR-GUA pair. There might be two possible interpretations for these phase delays: longitudinal propagation and latitudinal propagation of waves. However, if the longitudinal propagation is the case, the observational results should be interpreted as a wave propagating from LAQ to other stations. This is not plausible. Moreover, we can see that the phase difference between MSR and GUA is about $40^{\circ}$, though they are almost in the same longitude, implying the implausibility of longitudinal propagation. Instead, these observations may be interpreted as latitudinal propagation. The latitude of LAQ is $42.5^{\circ}$ which is higher than that of other stations, and this may cause the phase difference.

\subsection{Analysis of HER-KAK stations}

As we did at the end of the previous section, now we examine the phase differences of $\mathrm{Pi} 2$ pulsations in the northward direction between the HER (Hermanus, $-33.9^{\circ}$ ) and KAK stations for the three events that occurred on 7 December 1996, 4 January 1997, and 14 January 1997. Fig- 


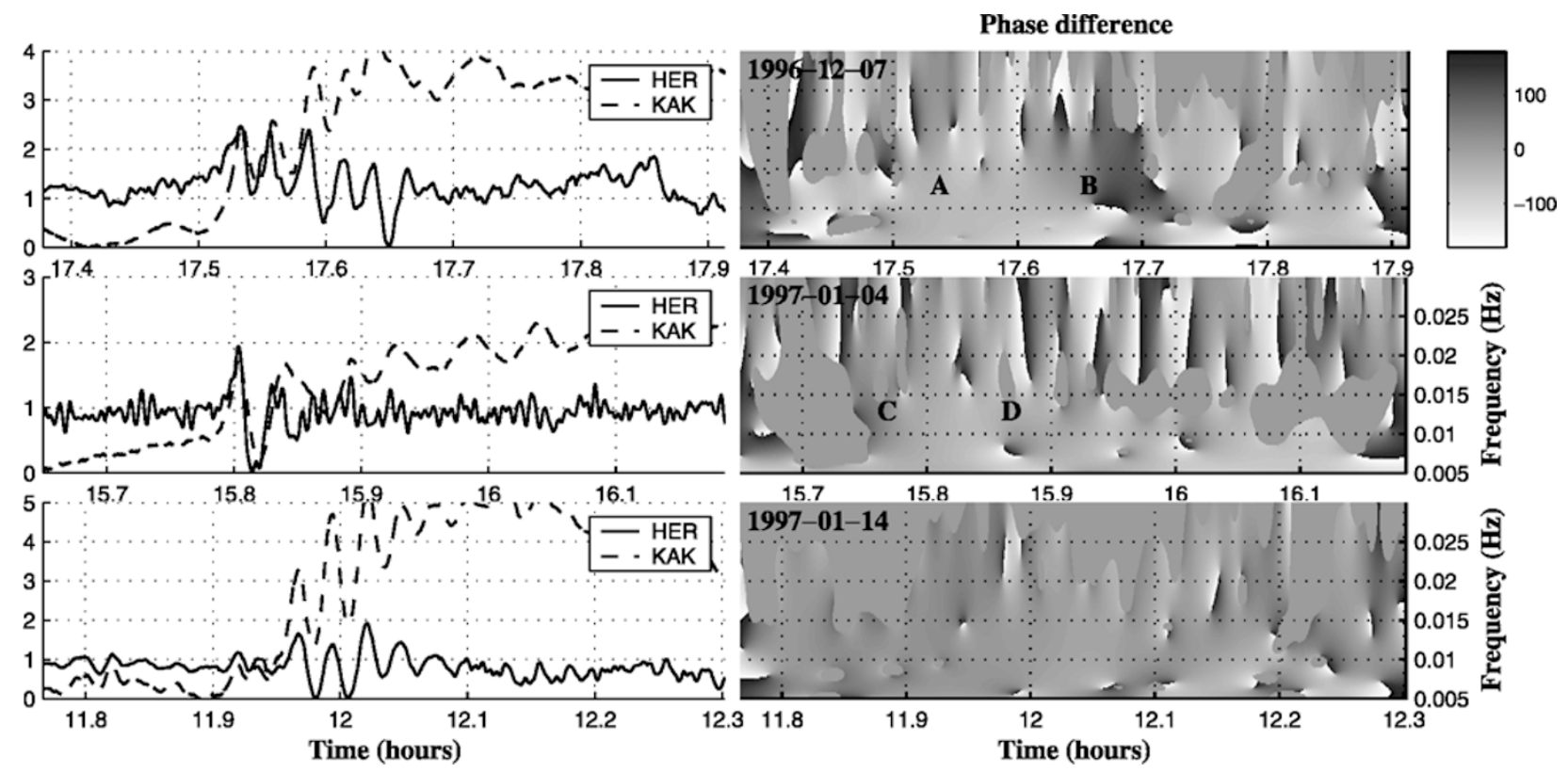

Fig. 9. North components of source signals and phase difference between HER and KAK stations for three events: 1996-12-07, 1997-01-04 and 1997-01-14.

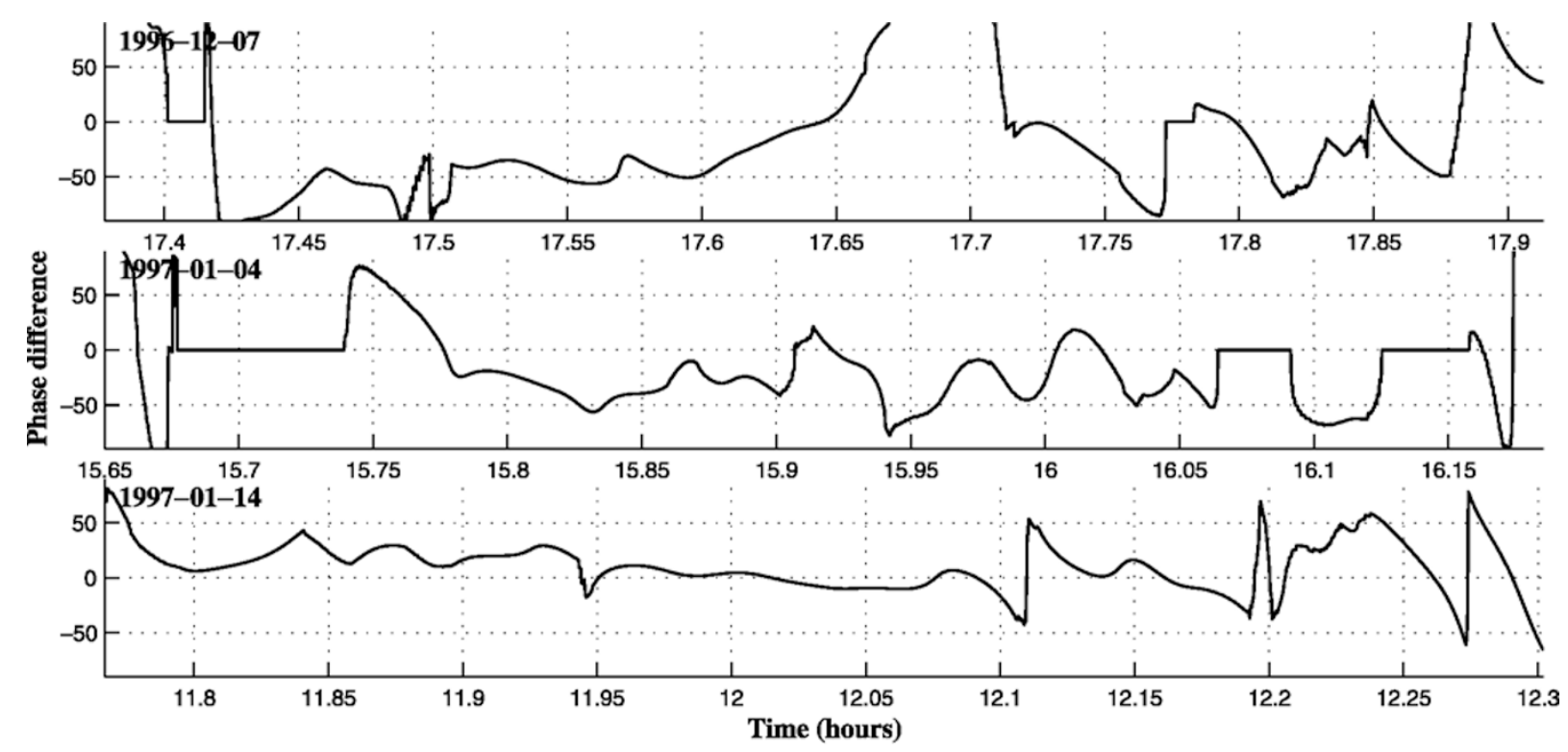

Fig. 10. Time-dependent phase differencies $\Delta \phi(t)$ averaged in the frequency band 0.01 and $0.015 \mathrm{~Hz}$.

ure 9 shows geomagnetic field variations recorded at HER and KAK on the left panel, and the results of polarization analysis on the right panel.

Similar to the derivation of frequency-dependent attributes in the previous section, one may also perform an averaging of the phase difference along the parameter $f$ (Fig. 10):

$$
\Delta \phi(t)=\frac{1}{\Delta F} \int_{F_{1}}^{F_{2}} \Delta \phi(t, \psi) d \psi
$$

On the left panel for the 14 January 1997 event, we almost do not observe any phase differences between the two stations during the event. $\mathrm{Pi} 2$ pulsations appeared in the time interval of 11.95-12.1 h, during which oscillation peaks were coincident between HER and KAK. This characteristic is reflected in the polarization analysis shown on the right panel. There are not significant changes in color, indicating no clear change in phase differences. The same behavior is also observed on the bottom panel of Fig. 10, which corresponds to this event.

However, in the first two events (the 7 December 1996 event and 4 January 1997 event) we can see apparent phase change during events. At the beginning of oscillations for the 1996-12-07 event (about 17.53 h, region " $A$ " on the topright panel in Fig. 9), phase difference is about $-50^{\circ}$, that we can read from the first panel of Fig. 10. After two to three cycles of oscillations, the phase difference becomes positive values, as indicated by the darker color in region 
"B". The 1997-01-04 event demonstrates an opposite behavior: at the beginning of oscillations the phase difference is slightly negative (region " $\mathrm{C}$ " on the middle-right panel in Fig. 9), but after one to two cycles it becomes a negative value of about $-50^{\circ}$ (region " $\mathrm{D}$ ") that can be identified by the change to a lighter color. A similar result can be also obtained from last two panels of Fig. 8, where we analyzed the phase difference for MSR-SMA and MSR-BLM pairs for the 20 September 1995 event.

It is difficult to explain the phase difference found on the 7 December 1996 and 4 January 1997 events by longitudinal propagation of waves from the substorm onset sector. This is because the oscillation at KAK lagged behind that at HER, even though KAK was located at nighttime and HER was on the afternoon side or the duskside. Instead, the phase difference may be explained in terms of the reflection coefficient of fast mode waves at the plasmapause. The plasmapause is not a perfect reflector for the fast mode waves, leading to leakage of the wave energy to the plasma trough (Fujita et al., 2000, 2002). If the condition of the plasmapause is different between the nightside and the afternoon side, the reflection rate of the fast mode waves at the plasmapause is expected to be different and may give rise to the phase shift between two stations. The no phase difference at very beginning stage of pulsation it may be explained by forcing oscillation due to the first pulse, which was propagating from the outer magnetosphere and supplies energy of the subsequent oscillations in the plasmasphere.

It may be possible to speculate that the phase change is related to the release of the magnetic energy in the magnetotail, which may be time-dependent and spatially inhomogeneous. Further studies using data from ground stations and satellites are needed to investigate causes of the phase change in more detail.

\section{Conclusions}

We propose a method for the analysis of polarization properties of geomagnetic field data using the continuous wavelet transform. The advantage of this method over previous techniques like Fourier analysis is that both the time and frequency dependence of the attributes can be obtained.

One important improvement by the new method is its ability to provide a means to investigate the time dependence of the phase difference. An important application of such information is in the determination of oscillation properties of $\mathrm{Pi} 2$ pulsation during the event, since it provides information on the excitation mechanism of $\mathrm{Pi} 2$ pulsations.

As shown in the presented examples, it is possible to extract all the attributes of the polarization ellipse. As a brief result of this polarization analysis, we determined that the phase difference may be somewhat sensitive to latitudes near the same MLT, and the imperfect reflecting plasmapause might be important in determining the local phase. In general, these conclusions should need further studied in a statistical manner. However, the techniques introduced in this paper could be useful for such future studies.
Acknowledgments. This project is supported by a grant from the Deutsche Forschungsgemeinschaft (DFG) within the framework of the priority program SPP 1114 "Mathematical methods for time series analysis and digital image processing". One of authors (MN) is supported by the Ministry of Education, Science, Sports, and Culture, Grant-in-Aid for Young Scientists (B) (grant 17740327) and the Kurata Memorial Hitachi Science and Technology Foundation (grant 844). We are especially grateful to Peter R. Sutcliffe (Hermanus Magnetic Observatory) for providing the experimental data set for HER station. We also thank the two anonymous reviewers for their helpful and constructive comments.

\section{References}

Diallo, M. S., M. Kulesh, M. Holschneider, F. Scherbaum, and F. Adler, Characterization of polarization attributes of seismic waves using continuous wavelet transforms, Geophysics, 71 (3), V67-V77, 2006.

Fujita, S., M. Itonaga, and H. Nakata, Relationship between the Pi2 pulsations and the localized impulsive current associated with the current disruption in the magnetosphere, Earth Planets Space, 52 (4), 267-281, 2000

Fujita, S., H. Nakata, M. Itonaga, A. Yoshikawa, and T. Mizuta, A numerical simulation of the $\mathrm{Pi} 2$ pulsations associated with the substorm current wedge, J. Geophys. Res., 107 (A3), 1034, 2002.

Han, D., T. Iyemori, Y. Gao, Y. Sano, F. Yang, W. Li, and M. Nosé, Local time dependence of the frequency of Pi2 waves simultaneously observed at 5 low-latitude stations, Earth Planets Space, 55 (10), 601-612, 2003.

Holschneider, M., Wavelets: an Analysis Tool, Clarendon Press, Oxford, 1995.

Kanasewich, E. R., Time Sequence Analysis in Geophysics, University of Alberta Press, Edmonton, Alberta, 1981.

Kosaka, K., T. Iyemori, M. Nosé, M. Bitterly, and J. Bitterly, Local time dependence of the dominant frequency of $\mathrm{Pi} 2$ pulsations at mid- and low-latitudes, Earth Planets Space, 54 (7), 771-781, 2002.

Lanzerotti, L. J. and L. V. Medford, Local night, impulsive (Pi2-type) hydromagnetic wave polarization at low latitudes, Planet. Space Sci., 32 (2), 135-142, 1984.

Lester, M., W. J. Hughes, and H. J. Singer, Polarization patterns of Pi2 magnetic pulsations and the substorm current wedgee, J. Geophys. Res., 88, 7958-7966, 1983.

Lester, M., W. J. Hughes, and H. J. Singer, Longitudinal structure in Pi2 pulsations and the substorm current wedge, J. Geophys. Res., 89, 54895494, 1984.

Morozov, I. B. and S. B. Smithson, Instantaneous polarization attributes and directional filtering, Geophysics, 61 (3), 872-881, 1996.

Nosé, M., Automated detection of Pi2 pulsations using wavelet analysis: 2. An application for dayside Pi2 pulsation study, Earth Planets Space, 51 (1), 23-32, 1999.

Nosé, M., K. Takahashi, T. Uozumi, K. Yumoto, Y. Miyoshi, A. Morioka, D. K. Milling, P. R. Sutcliffe, H. Matsumoto, T. Goka, and H. Nakata, Multipoint observations of a $\mathrm{Pi} 2$ pulsation on morningside: The 20 September 1995 event, J. Geophys. Res., 108 (A5), 1219, 2003.

Nosé, M., K. Liou, and P. R. Sutcliffe, Longitudinal dependence of characteristics of low-latitude Pi2 pulsations observed at Kakioka and Hermanus, Earth Planets Space, 58 (6), 775-783, 2006.

Osaki, H., K. Yumoto, K. Fukao, K. Shiokawa, F. W. Menk, B. J. Fraser, and the $210^{\circ} \mathrm{MM}$ Magnetic Observation Group, Characteristics of lowlatitude $\mathrm{Pi} 2$ pulsations along the $210^{\circ}$ magnetic meridian, J. Geomag. Geoelectr., 48, 1421-1430, 1996.

Pinnegar, C. R., Polarization analysis and polarization filtering of threecomponent signals with the time-frequency $\mathrm{S}$ transform, Geophys. $J$. Int., 165 (2), 596-606, 2006.

Rene, R. M., J. L. Fitter, P. M. Forsyth, K. Y. Kim, D. J. Murray, J. K. Walters, and J. D. Westerman, Multicomponent seismic studies using complex trace analysis, Geophysics, 51 (6), 1235-1251, 1986.

Yumoto, K., H. Osaki, K. Fukao, K. Shiokawa, Y. Tanaka, S. I. Solovyev, G. Krymskij, E. F. Vershinin, V. F. Osinin, and the $210^{\circ}$ MM Magnetic Oservation Group, Correlation of high- and low-latitude Pi2 magnetic pulsations observed at $210^{\circ}$ magnetic merdian chain stations, J. Geomag. Geoelectr., 46, 925-935, 1994.

M. Kulesh (e-mail: mkulesh@math.uni-potsdam.de), M. Nosé, M Holschneider, and K. Yumoto 